\title{
Liver Transplantation in Mixed Hepatocellular Carcinoma and Cholangiocarcinoma
}

\author{
Jong Man Kim \\ Department of Surgery, Samsung Medical Center, Sungkyunkwan University School of Medicine, Seoul, Korea
}

Received Mar. 11, 2019

Revised Apr. 29, 2019

Accepted Apr. 29, 2019
Mixed hepatocellular carcinoma and cholangiocarcinoma (HCC-CC) are rare tumors, and the risk factors associated with them are not well understood yet. Moreover, the diagnosis of mixed HCC-CC can be complicated due to the difficulty in distinguishing mixed HCC-CC from HCC and intrahepatic CCC on radiological images. Serum tumor markers are useful when the radiological images are inconclusive. It remains unclear whether the prognosis of mixed HCC-CC differs from that of HCC. However, several studies have reported that the tumor recurrence and patient survival rates of mixed HCC-CC were similar to those of HCC after liver transplantation (LT) and liver resection. In this paper, we report that LT in patients with mixed HCC-CC achieves outcomes which are similar to those seen in LT for HCC. Therefore, the diagnosis of mixed HCC-CC should not be considered as a contraindication for LT. (J Liver Cancer 2019;19:85-90)

Keywords: Liver transplantation; Liver malignancy; Outcomes

\section{INTRODUCTION}

Mixed hepatocellular carcinoma and cholangiocarcinoma (HCC-CC) comprise a minority of primary liver malignancies with histological features of both hepatocellular carcinoma (HCC) and cholangiocarcinoma (CC) ${ }^{1,2}$ The geographical distribution and the age and gender distribution for mixed HCC-CC and HCC are similar; however, mixed HCC-CC has a poor prognosis. ${ }^{3,4}$

The origin of mixed HCC-CC is still debatable; however, a bipotential precursor might explain the origin of mixed HCC-CC tumors. ${ }^{5}$ Patients often remain asymptomatic until

\section{Corresponding author : Jong Man Kim}

Department of Surgery, Samsung Medical Center, Sungkyunkwan University School of Medicine, 81 Irwon-ro, Gangnam-gu, Seoul 06351, Korea

Tel. +82-2-3410-1719, Fax. +82-2-3410-0040

E-mail; jongman94@hanmail.net

https://orcid.org/0000-0002-1903-8354 the advanced stage of the disease, and common symptoms, such as weight loss, malaise, abdominal discomfort, jaundice, hepatomegaly or a palpable abdominal mass, are non-specific. ${ }^{6}$ The original histological classification of mixed HCC-CC included three types: type A for separate nodules of HCC and intrahepatic CCC (iCCC), type B for contiguous masses that might mingle with continued growth, and type $\mathrm{C}$ for HCC and CC combined within the same tumor. ${ }^{7}$ A second classification system classified thee types; type I for coincidental occurrence of HCC and intrahepatic iCCC as separate nodules, type II for transitional tumors, and type III for the fibrolamellar HCC variant. ${ }^{7}$ In 2010, the WHO proposed a new classification for mixed HCC-CC: "classic" HCC-CC that includes Allen Type 1 and Good-man Type A tumors (distinct nodules of HCC and intrahepatic cholangiocarcinoma (ICC) ranging from low to high grade) $a^{2} d^{2}$ "stem cell” HCC-CC, which is further divided into three subgroups: typical, intermediate and cholangiocellular. ${ }^{3}$ 
The finding of mixed HCC-CC is much lower than that of HCC in liver transplantation (LT) patients (accounting for $0.4-14.2 \%$ of preoperatively diagnosed HCC patients) and a preoperative diagnosis is difficult. ${ }^{8}$ Most mixed HCC-CC lesions have been detected incidentally in surgical specimens. Moreover, because of its rarity, the clinic-pathological characteristics and post-transplant prognosis of mixed HCC-CC have not previously been reported in detail. Here, we review the literature and report the rates of misdiagnosis of mixed HCC-CC, and the outcomes after LT for mixed HCC-CC.

\section{DIAGNOSIS}

It is difficult to diagnose mixed HCC-CC accurately before surgical resection or LT (Table 1). Thus, most mixed HCCCC are misdiagnosed as HCC, and surgical resection or LT is performed. The overall incidence of misdiagnosed HCC or incidental ICC and/or mixed HCC-CC in patients undergoing LT for any cause was reported as $0.7 \%{ }^{9}$ The reported incidence of misdiagnosed iCCC alone was $0.34 \%$ and that of mixed HCC-CC alone was $0.48 \%(P=0.056)$. These findings suggest that mixed HCC-CC is more common than iCCC. In addition, the reported incidences of these uncommon tu- mors in liver explants were similar worldwide. ${ }^{9}$

While mixed HCC-CC tumors have been reported to be more common in male patients and in those with cirrhosis and/or chronic hepatitis, their clinical characteristics remain poorly understood. ${ }^{3}$ Some previous reports suggested that preoperative radiologic imaging with contrast enhancement in the arterial and portal venous phases without washout could reveal the presence of mixed HCC-CC. Several studies have reported that these tumors present characteristics of both iCCC and HCC but demonstrate an enhancement pattern and have ancillary features similar to iCCC. ${ }^{10,11}$ Additionally, these tumors might be associated with higher carbohydrate antigen (CA) 19-9 and alpha-fetoprotein levels. ${ }^{12}$ Therefore, distinguishing between mixed HCC-CC, HCC, and CCC without biopsy continues to be a challenge, and malignancies other than HCC are still encountered, often unexpectedly, in explanted liver specimens.

Dynamic contrast-enhanced magnetic resonance imaging (MRI) and computed tomography (CT) are often used in the evaluation of mixed HCC-CC. In one small case series study, MRI was reported to be $100 \%$ sensitive, while CT demonstrated a sensitivity of $78 \% .{ }^{13}$ The authors propose that the evidence of both HCC and CCC features in the same tumor

Table 1. Clinical characteristics of the patients in the selected studies

\begin{tabular}{|c|c|c|c|c|c|c|}
\hline Study & Country/study period & $\begin{array}{l}\text { Numbers of } \\
\text { mixed HCC-CC }\end{array}$ & $\begin{array}{c}\text { Mean/median } \\
\text { age (years) }\end{array}$ & $\begin{array}{l}\text { Median AFP } \\
\text { (ng/mL) }\end{array}$ & $\begin{array}{c}\text { Median CA 19-9 } \\
(\mathrm{U} / \mathrm{mL})\end{array}$ & $\begin{array}{l}\text { Mean/median } \\
\text { MELD score }\end{array}$ \\
\hline Gupta et al. ${ }^{9}$ & Japan/1996-2015 & $2 / 573(0.3)$ & 48.5 & $7(2-17)$ & $80(9-509)$ & 20 \\
\hline Serra et al. ${ }^{25}$ & Italy/2000-2015 & $4 / 655(0.6)$ & - & - & - & - \\
\hline Takahashi et al. ${ }^{26}$ & USA/2003-2014 & 4/1,188 (0.3) & 60.1 & - & - & 20 \\
\hline Itoh et al. ${ }^{22}$ & Japan/1999-2014 & $8 / 178(4.5)$ & 57.5 & $19.7(2.8-49.6)$ & $57.2(0.6-100.9)$ & - \\
\hline Sapisochin et al..$^{23}$ & Spain/2000-2010 & $24 / 7,876(3.0)$ & 58 & $6.6(1.2-216)$ & - & 11 \\
\hline Facciuto et al. ${ }^{21}$ & USA/1993-2013 & $25 / 3,073(0.8)$ & 60 & - & - & 14 \\
\hline Park et al. ${ }^{27}$ & Korea/1999-2009 & $15 / 2,137(0.7)$ & 59 & $32.6(0.9-793)$ & - & 14 \\
\hline Sapisochin et al." & USA/1999-2009 & 10/302 (3.3) & 59 & $6.5(1.6-464)$ & - & 16 \\
\hline Song et al. ${ }^{19}$ & Korea/1995-2012 & 8/- & 53.7 & - & - & 17 \\
\hline Panjala et al. ${ }^{1}$ & USA/1998-2008 & $12 /-$ & 61 & - & - & - \\
\hline Groeschl et al..$^{17}$ & SEER database/1973-2007 & $19 / 1,466(1.3)$ & 61.5 & - & - & - \\
\hline Jung et al. ${ }^{4}$ & Korea/2005-2014 & $32 / 3,103(1.0)$ & 53.4 & 32.6 & 13.7 & 14 \\
\hline
\end{tabular}

Values are presented as number (\%) or median (range).

HCC-CC, hepatocellular carcinoma and cholangiocarcinoma; AFP, alpha-fetoprotein; CA 19-9, carbohydrate antigen 19-9; MELD, model for endstage liver disease. 
suggest the possible presence of mixed HCC-CC. ${ }^{13}$ Another study reported that a high signal on $\mathrm{T} 2$ sequences, the presence of tumor areas with progressive enhancement/contrast retention, and lack of a capsule could indicate a mixed HCCCC. ${ }^{14}$ In 2013, Ijichi et al..$^{15}$ reported the use of ${ }^{18} \mathrm{~F}$-fluorodeoxyglucose positron emission tomography-CT (PET-CT) imaging to evaluate three patients by mixed HCC-CC. In this case series, all the mixed HCC-CC tumors were detected by PET, with maximum standardized uptake value (SUVmax) of 9.9, 12.0, and 13.0. Moreover, SUVmax levels correlated with the tumor size or tumor markers. Li et al. ${ }^{16}$ conducted a retrospective evaluation of tumor markers in their cohort of patients. They found a wide variety of characteristics displayed by mixed HCC-CC, particularly variations in alphafetoprotein (AFP) and CA 19-9 expression. They concluded that investigating patients for elevation of multiple tumor markers, or discordance between the tumor marker levels and imaging patterns of the lesions (i.e., AFP elevation but the absence of classical imaging features consistent with HCC), might significantly increase the diagnosis of mixed HCC-CC.

However, radiologists generally agree that it is extremely difficult to identify mixed HCC-CC accurately, based only on imaging findings. ${ }^{10}$ Therefore, several authors have proposed that lesion biopsy could be helpful for a more precise diagnosis. ${ }^{1}$

\section{OUTCOMES}

Survival of mixed HCC-CC patients depends on the type of treatment received. The most common treatment for mixed HCC-CC has been liver resection, and several studies have reported a 3-year survival rate of $25-50 \%$ after liver resection. ${ }^{4,17}$ HCC is now a primary indication for LT in patients with within Milan criteria or downstaged HCC, and an overall survival rate of $70 \%$ has been reported with $\mathrm{LT}^{4,7}$ Unfortunately, there are only a small number of studies about LT for mixed HCC-CC.

Chan et al. ${ }^{18}$ were the first to report about LT for mixed HCC-CC, and two of the three patients were alive with no evidence of disease at 25 and 35 months after the procedure.
Panjala et al. ${ }^{1}$ published the largest single-institution case series of 12 patients undergoing LT for mixed HCC-CC. Of these, one patient died 48 days after LT due to procedure-related complications, and the median overall survival (OS) of the remaining patients was 3.6 years. Song et al. ${ }^{19}$ reported outcomes in patients diagnosed with mixed HCC-CC either after liver resection for primary liver cancer (68 patients) or after LT for primary liver cancer (eight patients) between 1995 and 2012. In their study, one patient underwent deceased donor LT while seven patients underwent living donor LT. The authors reported that patients showed slightly better disease-free survival (DFS) and OS after LT, although the results were not statically significant. Specifically, 5 -year DFS rates were $26.2 \%$ vs. $37.5 \%$ ( $P=0.333$ ), while 5 -year OS rates were $42.1 \%$ vs. $50 \%(P=0.591)$. There were no differences in DFS or OS rates between the liver resection and LT group. Given the limitation due to the retrospective nature of the study, they concluded that the role of LT in the treatment of mixed HCC-CC remains unclear and that in cases with preserved liver function and tumors smaller than $5 \mathrm{~cm}$, liver resection should still be considered, particularly when complete resection with an adequate safety margin is possible. Jung et al. ${ }^{4}$ reported favorable post-transplant outcomes in patients with 1 or 2 mixed HCC-CCs $\leq 2.0 \mathrm{~cm}$ in size, with a tumor recurrence rate of $13.3 \%$ and a patient survival rate of $93.3 \%$ after 5 years. The long-term post-transplant prognoses were similar following LT and liver resection, but the post-recurrence patient survival rate was poor in LT recipients. These potential selection criteria for LT in mixed HCC-CC are similar to the super-selection criteria for LT in HCC patients, namely the presence of 1 or 2 tumors up to $2 \mathrm{~cm}$ in size. ${ }^{20}$ Considering the favorable outcomes reported for patients with 1 or 2 mixed HCC-CCs $\leq 2.0 \mathrm{~cm}$, this suggests that patients in the very early stage of mixed HCC-CC might be suitable candidates for LT. Additionally, given that very early mixed HCCCC demonstrated favorable post-transplant prognosis, less stricter follow-up may be required (Table 2).

Several studies have reported that the OS rate of mixed HCC-CC patients is comparable to that of HCC patients. ${ }^{11,21,22}$ However, one study reported that the OS rate of patients with HCC was statistically significantly higher than 
that of patients with iCCC or mixed HCC-CC. However, this difference was not significant in the subgroup analyses of patients with only mixed HCC-CC and in those with a solitary tumor less than $2 \mathrm{~cm}$ in size. ${ }^{23}$ Additionally, several studies found a significantly lower DFS in patients with iCCC or mixed HCC-CC compared to matched HCC patients; ${ }^{21,23}$ however, another study reported no difference in DFS between the two groups. ${ }^{22}$

A Spanish, matched cohort, multicenter study reported that patients with either iCCC or mixed HCC-CC had a higher rate of recurrence, a higher cumulative risk of recurrence, and a lower survival rate compared to HCC patients. ${ }^{23}$ However, further analyses demonstrated that these differences were driven by the iCCC patients in the group. Particularly, when we analyzed the iCCC and mixed HCC-CC subgroups separately, the mixed HCC-CC cohort had a 5-year survival rate of approximately $80 \%(n=15)$, equivalent to that of the matched cohort of HCC patients $(n=30)$. Furthermore, no significant differences were observed in the 5-year cumulative risk of recurrence between the mixed HCC-CC and HCC patients (7\% vs. $4 \%$, respectively). A recurrence rate was lower than what has been previously reported for mixed HCC-CC patients could be attributed to the fact that $67 \%$ of the patients had a single tumor, and $<20 \%$ showed microvascular invasion or satellite lesions. Further, they reported that mixed HCC-CC patients who had single tumors of size $2 \mathrm{~cm}$ or smaller, achieved excellent survival rates that were comparable to the controls. The mixed HCC-CC subgroup of patients that met the above criteria achieved a 5-year survival rate after LT, which is similar to that of HCC patients within the Milan criteria. ${ }^{24}$ The authors concluded that preoperative biopsy resulting in a diagnosis of mixed HCC-CC should not exclude patients from undergoing LT. ${ }^{23}$

Based on the data obtained from the United Network for Organ Sharing (UNOS) database collected between 1994 and 2013, patients who underwent LT for HCC-CC had overall 1 -, 3-, and 5-year survival rates of $82 \%, 47 \%$, and $40 \%$, respectively, and a median survival duration of 29 months. ${ }^{7}$ Patients who underwent LT for HCC had significantly better 1 -, 3-, and 5-year survival rates $(86 \%, 72 \%$, and 62\%, respectively) than those who underwent LT for mixed HCC-CC. ${ }^{7}$ Additionally, LT for mixed HCC-CC achieves a survival rate similar to that after LT in carefully selected patients of iCCC, based on the data from the UNOS database. Similarly, graft survival rate was significantly better in the HCC group (82\%, $68 \%, 54 \%$ at 1-, 3-, and 5-years, respectively) compared to patients with mixed HCC-CC or iCCC. ${ }^{7}$ Interestingly, the acute rejection rate at 6 months was higher in patients with

Table 2. Pathological data and outcomes

\begin{tabular}{|c|c|c|c|c|c|c|c|c|c|}
\hline \multirow{2}{*}{ Study } & \multirow{2}{*}{$\begin{array}{l}\text { Mean/median } \\
\text { tumor size }(\mathrm{cm})\end{array}$} & \multirow{2}{*}{$\begin{array}{c}\text { Mean/ } \\
\text { median tumor } \\
\text { numbers }\end{array}$} & \multirow{2}{*}{$\begin{array}{l}\text { Mean/median } \\
\text { follow-up durations } \\
\text { (months) }\end{array}$} & \multicolumn{3}{|c|}{ Disease-free survival rate (\%) } & \multicolumn{3}{|c|}{ Overall survival rate (\%) } \\
\hline & & & & 1-year & 3-year & 5-year & 1-year & 3-year & 5-year \\
\hline Gupta et al. ${ }^{9}$ & 1.8 & 1 & $60(6-168)$ & 60 & 60 & 60 & 60 & 60 & 60 \\
\hline Serra et al. ${ }^{25}$ & - & - & - & - & - & - & - & - & - \\
\hline Takahashi et al. ${ }^{26}$ & 2.1 & 1.9 & 18.8 & 67 & 42 & & - & - & - \\
\hline Itoh et al. ${ }^{22}$ & 2.6 & - & - & 86 & 86 & 86 & 88 & 73 & 73 \\
\hline Sapisochin et al. ${ }^{23}$ & 2.9 & 1 & $41.7(3.3-140.6)$ & - & - & - & 83 & 70 & 60 \\
\hline Facciuto et al. ${ }^{21}$ & 2.5 & 1 & 47 & 62 & - & 44 & 71 & - & 57 \\
\hline Park et al. ${ }^{27}$ & 2.9 & - & - & 60 & 53 & 53 & 66.7 & 60 & 60 \\
\hline Sapisochin et al." & 3 & 1 & 32 & 60 & - & 30 & 79 & - & 32 \\
\hline Song et al..$^{19}$ & 2.1 & 3 & $48.6(11-124)$ & 50 & 38 & 25 & 75 & 50 & 25 \\
\hline Panjala et al.' & - & - & 111.6 & - & - & - & 79 & 66 & 16 \\
\hline Groeschl et al. ${ }^{17}$ & - & - & $36(19-89)$ & - & - & - & 89 & 48 & - \\
\hline Jung et al. ${ }^{4}$ & 2.5 & 1.3 & 48.6 & - & - & - & 84.4 & 73.1 & 65.8 \\
\hline
\end{tabular}

Values are presented as number (range) unless otherwise indicated. 
iCCC $(22.4 \%)$ than in those with mixed HCC-CC (19\%) and HCC (12.7\%). ${ }^{7}$ The recurrence rate in mixed HCC-CC patients after LT was reported to be $42 \%$ in a meta-analysis. ${ }^{9}$ The most common site for recurrence was extrahepatic (73\%). Intrahepatic recurrence was noted in $12 \%$ of all recurrences, while both intra- and extrahepatic recurrences were present in $15 \%$ of recurrence cases.

\section{CONCLUSIONS}

Surgical resection is associated with acceptable outcomes in mixed HCC-CC. In addition, the long-term outcomes of tumor recurrence and patient survival following LT and liver resection were similar in patients with mixed HCC-CC and HCC. Therefore, if mixed HCC-CC is diagnosed on biopsy, the patient should not be excluded from the LT program. However, reported studies have been limited by small sample sizes and do not allow for definite conclusions. Future prospective studies are required to maximize the benefits of LT in patients with mixed HCC-CC.

\section{Conflicts of Interest}

The authors have no conflicts to disclose.

\section{REFERENCES}

1. Panjala C, Senecal DL, Bridges MD, Kim GP, Nakhleh RE, Nguyen $\mathrm{JH}$, et al. The diagnostic conundrum and liver transplantation outcome for combined hepatocellular-cholangiocarcinoma. Am J Transplant 2010;10:1263-1267.

2. Wang J, Wang $F$, Kessinger $A$. Outcome of combined hepatocellular and cholangiocarcinoma of the liver. J Oncol 2010;2010:917356.

3. Magistri P, Tarantino G, Serra V, Guidetti C, Ballarin R, Di Benedetto F. Liver transplantation and combined hepatocellular-cholangiocarcinoma: feasibility and outcomes. Dig Liver Dis 2017;49:467470.

4. Jung DH, Hwang S, Hong SM, Chung YK, Song GW, Lee YJ, et al. Post-resection prognosis of combined hepatocellular carcinomacholangiocarcinoma according to the 2010 WHO classification. World J Surg 2017;41:1347-1357.

5. Spagnoli FM, Amicone L, Tripodi M, Weiss MC. Identification of a bipotential precursor cell in hepatic cell lines derived from transgenic mice expressing cyto-Met in the liver. J Cell Biol
1998;143:1101-1112.

6. Zhang H, Yang T, Wu M, Shen F. Intrahepatic cholangiocarcinoma: epidemiology, risk factors, diagnosis and surgical management. Cancer Lett 2016;379:198-205.

7. Vilchez V, Shah MB, Daily MF, Pena L, Tzeng CW, Davenport D, et al. Long-term outcome of patients undergoing liver transplantation for mixed hepatocellular carcinoma and cholangiocarcinoma: an analysis of the UNOS database. HPB (Oxford) 2016;18:29-34.

8. Lee SD, Park SJ, Han SS, Kim SH, Kim YK, Lee SA, et al. Clinicopathological features and prognosis of combined hepatocellular carcinoma and cholangiocarcinoma after surgery. Hepatobiliary Pancreat Dis Int 2014;13:594-601.

9. Gupta R, Togashi J, Akamatsu N, Sakamoto Y, Kokudo N. Impact of incidental/misdiagnosed intrahepatic cholangiocarcinoma and combined hepatocellular cholangiocarcinoma on the outcomes of liver transplantation: an institutional case series and literature review. Surg Today 2017;47:908-917.

10. Fowler KJ, Sheybani A, Parker RA 3rd, Doherty S, M Brunt E, Chapman WC, et al. Combined hepatocellular and cholangiocarcinoma (biphenotypic) tumors: imaging features and diagnostic accuracy of contrast-enhanced CT and MRI. AJR Am J Roentgenol 2013;201:332-339.

11. Sapisochin G, Fidelman N, Roberts JP, Yao FY. Mixed hepatocellular cholangiocarcinoma and intrahepatic cholangiocarcinoma in patients undergoing transplantation for hepatocellular carcinoma. Liver Transpl 2011;17:934-942.

12. Kim KH, Lee SG, Park EH, Hwang S, Ahn CS, Moon DB, et al. Surgical treatments and prognoses of patients with combined hepatocellular carcinoma and cholangiocarcinoma. Ann Surg Oncol 2009;16:623-629.

13. Shetty AS, Fowler KJ, Brunt EM, Agarwal S, Narra VR, Menias CO. Combined hepatocellular-cholangiocarcinoma: what the radiologist needs to know about biphenotypic liver carcinoma. Abdom Imaging 2014;39:310-322.

14. de Campos RO, Semelka RC, Azevedo RM, Ramalho M, Heredia V, Armao DM, et al. Combined hepatocellular carcinoma-cholangiocarcinoma: report of MR appearance in eleven patients. J Magn Reson Imaging 2012;36:1139-1147.

15. Ijichi H, Shirabe K, Taketomi A, Yoshizumi T, Ikegami T, Mano Y, et al. Clinical usefulness of (18) F-fluorodeoxyglucose positron emission tomography/computed tomography for patients with primary liver cancer with special reference to rare histological types, hepatocellular carcinoma with sarcomatous change and combined hepatocellular and cholangiocarcinoma. Hepatol Res 2013;43:481487.

16. Li R, Yang D, Tang CL, Cai P, Ma KS, Ding SY, et al. Combined hepatocellular carcinoma and cholangiocarcinoma (biphenotypic) tumors: clinical characteristics, imaging features of contrast- 
enhanced ultrasound and computed tomography. BMC Cancer 2016;16:158.

17. Groeschl RT, Turaga KK, Gamblin TC. Transplantation versus resection for patients with combined hepatocellular carcinomacholangiocarcinoma. J Surg Oncol 2013;107:608-612.

18. Chan AC, Lo CM, Ng IO, Fan ST. Liver transplantation for combined hepatocellular cholangiocarcinoma. Asian J Surg 2007;30:143-146.

19. Song S, Moon HH, Lee S, Kim TS, Shin M, Kim JM, et al. Comparison between resection and transplantation in combined hepatocellular and cholangiocarcinoma. Transplant Proc 2013;45:30413046.

20. Hwang S, Ahn CS, Kim KH, Moon DB, Ha TY, Song GW, et al. Super-selection of a subgroup of hepatocellular carcinoma patients at minimal risk of recurrence for liver transplantation. J Gastrointest Surg 2011;15:971-981.

21. Facciuto $M E$, Singh $M K$, Lubezky N, Selim MA, Robinson D, KimSchluger $L$, et al. Tumors with intrahepatic bile duct differentiation in cirrhosis: implications on outcomes after liver transplantation. Transplantation 2015;99:151-157.

22. Itoh S, Ikegami T, Yoshizumi T, Wang H, Takeishi K, Harimoto N, et al. Long-term outcome of living-donor liver transplantation for combined hepatocellular-cholangiocarcinoma. Anticancer Res
2015;35:2475-2476.

23. Sapisochin G, de Lope CR, Gastaca M, de Urbina JO, LópezAndujar R, Palacios F, et al. Intrahepatic cholangiocarcinoma or mixed hepatocellular-cholangiocarcinoma in patients undergoing liver transplantation: a Spanish matched cohort multicenter study. Ann Surg 2014;259:944-952.

24. Mazzaferro V, Llovet JM, Miceli R, Bhoori S, Schiavo M, Mariani L, et al. Predicting survival after liver transplantation in patients with hepatocellular carcinoma beyond the Milan criteria: a retrospective, exploratory analysis. Lancet Oncol 2009;10:35-43.

25. Serra V, Tarantino G, Guidetti C, Aldrovandi S, Cuoghi M, Olivieri $T$, et al. Incidental intra-hepatic cholangiocarcinoma and hepatocholangiocarcinoma in liver transplantation: a single-center experience. Transplant Proc 2016;48:366-369.

26. Takahashi K, Obeid J, Burmeister CS, Bruno DA, Kazimi MM, Yoshida A, et al. Intrahepatic cholangiocarcinoma in the liver explant after liver transplantation: histological differentiation and prognosis. Ann Transplant 2016;21:208-215.

27. Park YH, Hwang S, Ahn CS, Kim KH, Moon DB, Ha TY, et al. Long-term outcome of liver transplantation for combined hepatocellular carcinoma and cholangiocarcinoma. Transplant Proc 2013:45:3038-3040. 